05

\title{
Сравнение СТМ и АСМ измерений тонких пленок Мо с моделью Кардара-Паризи-Жанга
}

\author{
() Л.А. Фомин, ${ }^{1}$ И.В. Маликов, ${ }^{1}$ В.А. Березин, ${ }_{1}^{1}$ А.Э. Рассадин, ${ }^{2}$ А.Б. Логинов, ${ }^{3}$ Б.А. Логинов ${ }^{4}$ \\ ${ }^{1}$ Институт проблем технологии микроэлектроники и особо чистых материалов РАН, \\ 142432 Черноголовка, Московская обл., Россия \\ ${ }^{2}$ Национальный исследовательский университет „Высшая школа экономики“, \\ 603014 Нижний Новгород, Россия \\ ${ }^{3}$ Московский государственный университет им. М.В. Ломоносова, \\ 119991 Москва, Россия \\ ${ }^{4}$ Национальный исследовательский университет МИЭТ, \\ 124498 Москва, Россия \\ e-mail: fomin@iptm.ru
}

Поступило в Редакцию 29 апреля 2021 г.

В окончательной редакции 29 апреля 2021 r.

Принято к публикации 29 апреля 2021 г.

Методами сканирующей туннельной микроскопии и атомно-силовой микроскопии исследован рельеф эпитаксиальных пленок Мо малых толщин, выращенных на $R$-плоскости сапфира. Найдена область параметров модели эволюции рельефа поверхности пленок Кардара-Паризи-Жанга, в которой она соответствует полученным экспериментальным результатам.

Ключевые слова: эпитаксиальные пленки, тугоплавкие металлы, шероховатая поверхность, принцип максимума Олейник-Лакса, преобразование Коула-Хопфа.

DOI: $10.21883 / J T F .2021 .10 .51358 .130-21$

\section{Введение}

В настоящее время применяемые в микроэлектронике для межсоединений и шин металлизации алюминий и медь с уменышением размеров элементов и возрастанием плотности тока в них перестают удовлетворять современным требованиям [1,2]. Низкие температуры плавления этих металлов ограничивают их применение, поскольку вызванные электромиграцией процессы деградации, приводящие к отказам, активируются с ростом отношения рабочей температуры к температуре плавления. Одним из методов решения указанной проблемы является использование металлов с низкой электромиграцией, в том числе тугоплавких металлов. При малых размерах проводников вклад в сопротивление рассеяния электронов проводимости на поверхности становится доминирующим при температурах вплоть до комнатной [2]. Экспериментальных данных по получению и исследованию проводимости сверхтонких пленок металлов и, тем более, влиянию морфологии поверхности на сопротивление для пленок металлов сверхмалых толщин относительно мало, что, по-видимому, связано со сложностью их получения и характеризации, а также малой устойчивостью таких пленок к окислению.

Ранее нами [3] были выращены эпитаксиальные тонкие пленки молибдена на $R$-плоскости сапфира методом импульсного лазерного осаждения в сверхвысоком вакууме по разработанной технологии. Во многих известных работах, например [4,5], морфология поверхности исследовалась методом сканирующей туннельной микроскопии (СТМ). Нами были проведены АСМ и
СТМ измерения образцов, направленные на исследование статистических свойств поверхности. Результаты сравнивались с известными моделями влияния шероховатости поверхности пленок металлов на их электроннотранспортные свойства. Полученные экспериментальные результаты показали квадратичную размерную зависимость проводимости $\sigma$ от толщины пленки при комнатной температуре и кубическую при низкой температуре. Такие сильные толщинные зависимости указывают на то, что рассеяние электронов на дефектах поверхности велико. Однако, как показывает эксперимент [3], корреляционная длина неровностей рельефа поверхности велика по сравнению с фермиевской длиной волны электронов $\left(L_{c} / \lambda_{F} \gg 1\right)$, из-за чего рассеяние на поверхностных дефектах должно быть малым. Полученное противоречие можно разрешить, предположив, что рельеф поверхности пленки близок к фрактальному. Фрактальность рельефа эпитаксиально растущей пленки следует из моделей эволюции рельефа поверхности, в которых на его формирование сильно влияют флуктуации потока атомов. Одной из наиболее известных такого типа является модель Кардара-Паризи-Жанга (КПЖ) [6]. Помимо этого, сравнение результатов СТМ и АСМ измерений в работе [3] показало значительную (в несколько раз) разницу в среднеквадратичных шероховатостях и корреляционных длинах, полученных этими двумя методами.

В настоящей работе были проведены дополнительные исследования рельефа пленок малых толщин методами СТМ и АСМ. Экспериментальные результаты сравнивались моделью эволюции рельефа поверхности пленок КПЖ. 


\section{1. Модель эволюции рельефа поверхности КПЖ}

Одной из наиболее известных моделей эволюции рельефа поверхности пленок в процессе роста является феноменологическая модель КПЖ [6], которая основывается на уравнении

$$
\frac{\partial H}{\partial t}=c \sqrt{1+(\nabla H)^{2}}+v \nabla^{2} H+\eta,
$$

где $H$ - локальная высота, $\nabla$ - двумерный градиент, $t$ - время. Несмотря на то что модель КПЖ появилась уже достаточно давно, она продолжает оставаться в фокусе интенсивных исследований [7-11]. В этой модели принимаются во внимание два конкурирующих процесса: рост поверхности пленки по локальной нормали к самой себе со скоростью с и ее стягивание силами поверхностного натяжения (второй член, $v \nabla^{2} H$ ), так, чтобы уменьшить площадь. Второй член также можно истолковывать как описывающий процесс десорбции атомов с поверхности $[11,12]$. При этом диффузия атомов осаждающегося вещества по поверхности в уравнении (1) не учитывается, и может быть учтена добавлением дополнительного члена с пространственными производными четвертого порядка $[12,13]$. Последний член, $\eta-$ в уравнении - флуктуационный, связанный с неравномерностью потока атомов испаряемой мишени на подложку. Например, для используемого метода лазерной абляции характерна флуктуация энергии излучения от импульса к импульсу. Кроме того, сам импульсный процесс испарения подразумевает неравномерность, связанную как с периодичностью импульсного испарения, так и с влиянием рельефа мишени, проявляющегося в самом процессе абляции. Причем рельеф мишени влияет именно на пространственные флуктуации потока осаждаемого материала.

Для флуктуационного члена принимается, что он дельта-коррелирован по пространству и времени:

$$
\langle\eta(x, t)\rangle=0,\left\langle\eta(x, t) \eta\left(x^{\prime}, t^{\prime}\right)\right\rangle=2 D \delta\left(x-x^{\prime}\right) \delta\left(t-t^{\prime}\right) .
$$

Здесь $2 D$ - дисперсия флуктуаций [12].

Уравнение (1) можно упростить, рассмотрев малоугловое приближение: $|\nabla H| \ll 1$, которое всегда выполняется для гладких пленок и было применено в оригинальной работе [6]. Тогда после разложения входящего в правую часть этого уравнения квадратного корня в ряд Тейлора получим

$$
\frac{\partial H}{\partial t}=c+\frac{c}{2}(\nabla H)^{2}+v \nabla^{2} H+\eta .
$$

Для уравнения (3) было получено точное решение [14] в пренебрежении третьим и четвертым членами, что означает, что десорбцией, поверхностной диффузией и флуктуациями пренебрегалось. Это решение в момент времени $t$ с помощью метода характеристик [14] в неявном виде записывается

$$
\begin{gathered}
x=y-c t \nabla H_{0}(y), \quad y \in \Omega_{0}, \\
H=H(y, t),
\end{gathered}
$$

где

$$
H(y, t)=c t+H_{0}(y)-\frac{c t}{2}\left(\nabla H_{0}(y)\right)^{2} .
$$

Первый член в формуле (6) выражает однородный рост высоты пленки. Однако эта формула справедлива только до некоторого момента времени $t^{*}$ - до наступления так называемой градиентной катастрофы [14], когда два фронта роста набегают друг на друга и в точке их соприкосновения решение становится неоднозначным. После же наступления градиентной катастрофы правильное решение представляет собой огибающую фронтов роста, которую можно построить с помощью принципа абсолютного максимума Олейник-Лакса [15]. Согласно этому принципу, рассматривается следующая функция:

$$
G(y ; x, t)=H_{0}(y)-\frac{(y-x)^{2}}{2 c t},
$$

экстремум которой определяется условием

$$
\nabla_{y} G(y ; x, t)=\nabla H_{0}(y)-\frac{(y-x)}{c t}=0,
$$

что совпадает с формулой (4). Пусть $y_{w}(x, t)-$ точка абсолютного максимума функции (7) при фиксированных $x$ и $t$, которая найдена каким-либо методом:

$$
y_{w}(x, t)=\arg \max _{y \in \Omega_{0}} G(y, x, t) .
$$

Тогда, согласно принципу Олейник-Лакса, приведенная высота поверхности $h(x, t)=H(x, t)-c t$ равна

$$
\begin{aligned}
h(x, t)= & H_{0}\left(y_{w}(x, t)\right)-\frac{\left(y_{w}(x, t)-x\right)^{2}}{2 c t} \\
& \equiv G\left(y_{w}(x, t) ; x, t\right) .
\end{aligned}
$$

В результате численного моделирования, согласно формуле (10), проведенного в работе [13], при котором в качестве начального условия брался экспериментально (методом АCM) измеренный рельеф подложки сапфира, было получено, что с течением времени среднеквадратичная шероховатость поверхности растущей пленки уменьшается, фрактальная размерность также уменьшается, а корреляционная длина увеличивается.

Другой способ решения уравнения (3) без учета флуктуационного члена, но с учетом поверхностного натяжения - сведение его к уравнению диффузии с помощью преобразования Коула-Хопфа [14]:

$$
H=c t+\frac{2 v}{c} \log (\varphi) .
$$


Тогда уравнение (3) без флуктуационного члена приобретает вид

$$
\frac{\partial \varphi}{\partial t}=v \nabla^{2} \varphi
$$

Решение задачи Коши для этого уравнения известно и записывается в виде интеграла

$$
\begin{aligned}
& \varphi(x, t)=\frac{1}{4 \pi v t} \\
& \times \iint \exp \left(\frac{1}{2 v t}\left[h_{0}\left(x_{1}\right) c t-\frac{\left(x-x_{1}\right)^{2}}{2}\right]\right) d^{2} x_{1},
\end{aligned}
$$

откуда по формуле (11) вычисляется $H$.

Аналитическое решение уравнения (3) в присутствии флуктуационного члена, насколько нам известно, в двумерном случае не найдено.

\section{2. Методики экспериментов}

Пленки Мо с толщинами 3, 6 и $19 \mathrm{~nm}$ были выращены на $R$-плоскости сапфира методом импульсного лазерного осаждения в сверхвысоком вакууме при температуре $450^{\circ} \mathrm{C}$ (см. [3] и ссылки там). Для получения пленок использовались специально отобранные подложки с шероховатостью около $0.2 \mathrm{~nm}$. Рельеф подложек измерялся методом АСМ. Типичный рельеф подложки и его двумерная автокорреляционная функция показаны в работе [16]. Для исследований микрорельефа пленок Мо использовался прибор „СММ-2000“ (завод ПРОТОН, г. Зеленоград). Измерения проводились на воздухе в течение одной недели после получения пленок. При этом образцы хранились при комнатной температуре в специальном закрытом бюксе с силикагелем.

В режиме СТМ зондом являлась качественно изготовленная из волоченной платиновой проволоки диаметром $0.2 \mathrm{~mm}$ игла, с контролем одноострийности и наличия на конце не более одного выступающего атома методом снятия кривой отвода с десятикратным спадом туннельного тока при отводе иглы на расстояние не более $0.2 \mathrm{~nm}$. Такие иглы позволяют достигать разрешение до $0.02 \mathrm{~nm}$ по латерали и высотам, с качественной визуализацией атомов в условии сверхвысокого вакуума. Измерения проводились в режиме поддержания фиксированного тока, когда в каждой точке игла зависает, так, чтобы ток был постоянным и равным назначенному, в нашем случае $4 \mathrm{nA}$. Расстояние обычно между СТМ иглой и образцом составляло около $1 \mathrm{~nm}$.

В качестве зондов в режиме АСМ использованы серийные кантилеверы с рекордно малым радиусом закругления острия $2 \mathrm{~nm}$ марки „MSNL“ фирмы Bruker, США, позволяющие достигать разрешение в $2 \mathrm{~nm}$ по латеральным размерам и до $0.05 \mathrm{~nm}$ по высотам рельефа. Также АСМ измерения выполнялись на микроскопе P47-Solver (НТ-МДТ, Москва, Зеленоград) с использованием кантилеверов TipsNano (Москва, Зеленоград). $\mathrm{ACM}$ измерения проводились в контактном режиме.

\section{3. Экспериментальные результаты}

Одной из причин, по которой сложно исследовать пленки малых и сверхмалых толщин, является их быстрая деградация из-за процессов окисления за времена сравнимые с временами, необходимыми для проведения анализа ex-situ. Для пленок Мо ситуация оказывается более оптимистичной. Проведенные измерения зависимости от времени сопротивления тонких $\sim 2.5 \mathrm{~nm}$ эпитаксиальных пленок Мо показали, что окисление происходит сравнительно медленно, и пленки Мо нанометровых толщин мало меняют свои электрические свойства в течение часов, дней и недель.

Изменение сопротивления со временем связано с уменьшением толщины проводящего слоя пленки из-за уменьшения его толщины. А плавное изменение электрофизических свойств пленок сверхтонких Мо позволяет предположить, что их окисление происходит изотропно, что также подтверждается логарифмической зависимостью изменения толщины (в нашем случае электрического сопротивления) пленок от времени, являющейся характерной для процессов окисления металлов, в том числе массивных образцов. Известно, что Мо устойчив к окислению при комнатной температуре, однако, поскольку сверхтонкие эпитаксиальные пленки являются неравновесным объектом и могут иметь остаточные напряжения, возникающие в процессе гетероэпитаксии, всегда возникает вопрос их резистентности к окислению, как структур неравновесных. В нашем случае оценочная толщина окисленного слоя Мо составила величину менее $0.3 \mathrm{~nm}$ за $300 \mathrm{~h}$ нахождения на воздухе при комнатной температуре при оценке изменения сопротивлению с толщиной по линейному закону. При реально наблюдающейся для таких толщин пленок Мо суперквадратичной зависимости сопротивления от толщины пленки оцениваемая толщина окисленного слоя будет еще меньше. Все это позволяет производить исследование таких пленок в течение разумных промежутков времени (часов и дней).

На рис. 1 показаны АСМ и СТМ сканы размерами $1000 \times 1000 \mathrm{~nm}$ поверхности пленок Мо с толщинами 3 , 6 и $19 \mathrm{~nm}$. Видно, что изображения, полученные с помощью двух разных методик, для одной и той же пленки похожи. Разница в углах наклона террас по отношению к оси х на изображениях обусловлена лишь тем, что образец ставился под разными углами. Латеральный размер террас по виду совпадает, что означает отсутствие явных артефактов при измерениях.

По результатам математической обработки определялись параметры поверхности, как визуально представлено на рис. 2, где схематично изображен профиль поверхности. Из рисунка видно, что существуют особенности большого масштаба (обозначенные $\mathrm{Sm}$ ) и малого масштаба (обозначенные $S$ ). При этом $S m-$ среднее от расстояний по горизонтали $S m_{1}, S m_{2}$ и т.д. между пересечениями средней линии этим профилем, 

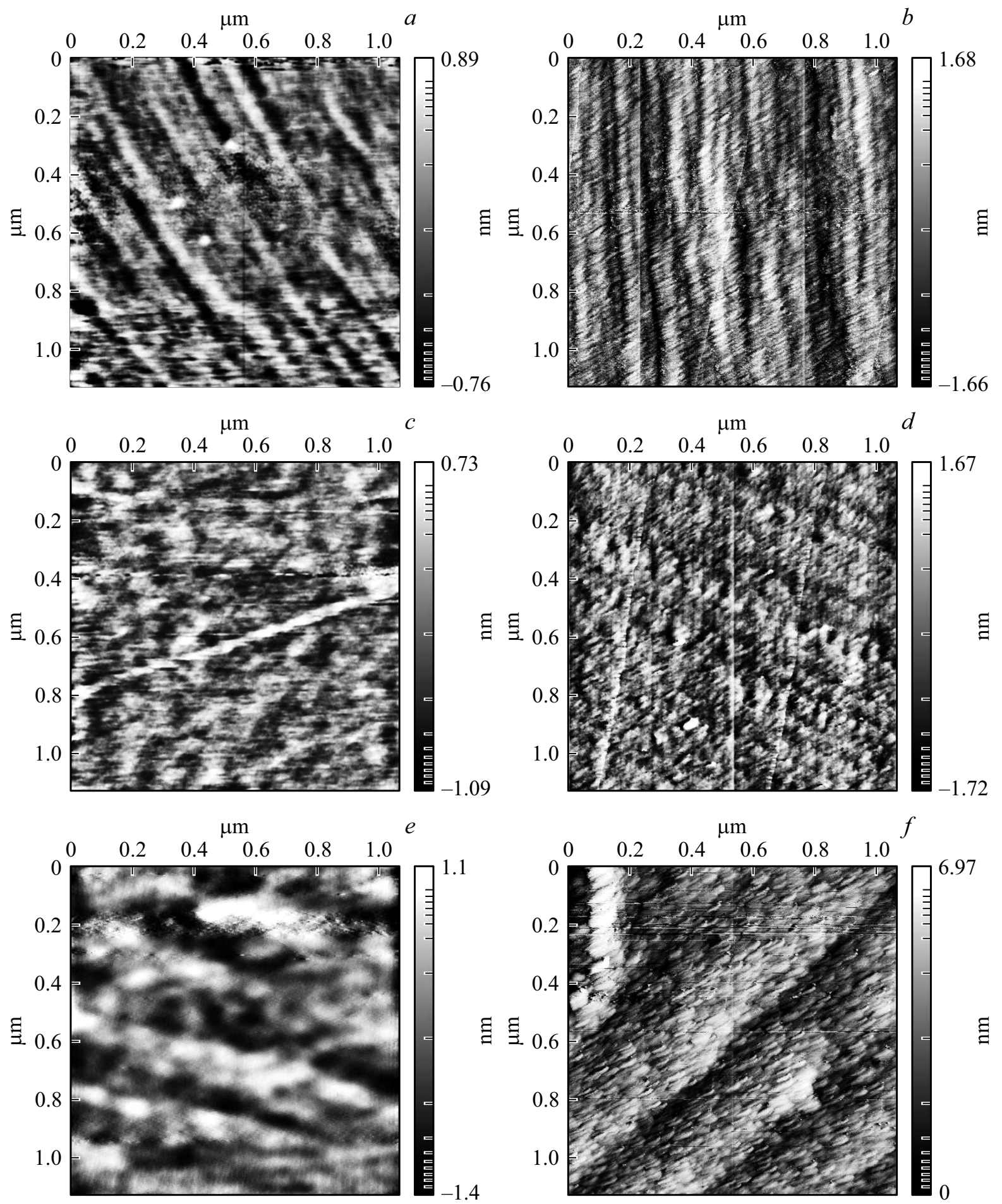

Рис. 1. $\mathrm{ACM}(a, c, e)$ и $\mathrm{CTM}(b, d, f) 1000 \times 1000 \mathrm{~nm}$ изображения поверхности пленок Мо с толщинами $3(a, b)$, $6(c, d)$ и $19(e, f) \mathrm{nm}$, выращенных на $R$-плоскости сапфира.

a $S$ - среднее от расстояний по горизонтали между локальными максимумами $S_{1}, S_{2}$ и т.д. профиля.

На рис. 3 показан пример статистического анализа профилей СТМ и АСМ изображений рельефа поверхности пленки Мо толщиной $19 \mathrm{~nm}$, произведенного программой микроскопа СММ-2000 по стандартным методикам. Как видно из рисунка, в результате статисти- ческого анализа АCM изображения величина Sm составляет $159.7 \mathrm{~nm}$. Вместе с тем статистический анализ СТМ изображения показывает, что эта величина составляет $32.5 \mathrm{~nm}$.

В табл. 1 сведены значения статистических параметров рельефа пленок в зависимости от толщины. Как видно из таблицы, величина среднеквадратичного откло- 


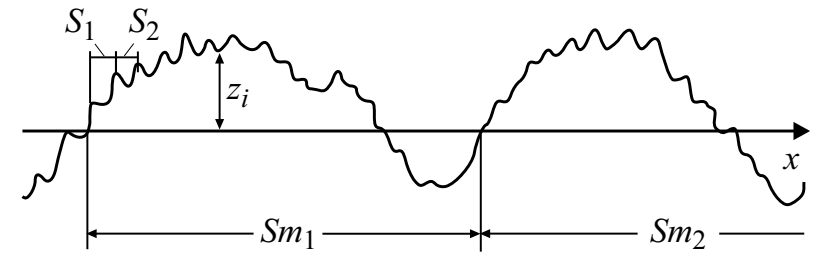

Рис. 2. Параметры шероховатости: $S m-$ среднее от расстояний по горизонтали между пересечениями $S m_{1}, S m_{2} \ldots$ профилем средней линии при ходе профиля вверх; $S$ - среднее от расстояний по горизонтали между максимумами $S_{1}, S_{2} \ldots$ профиля.

$a$

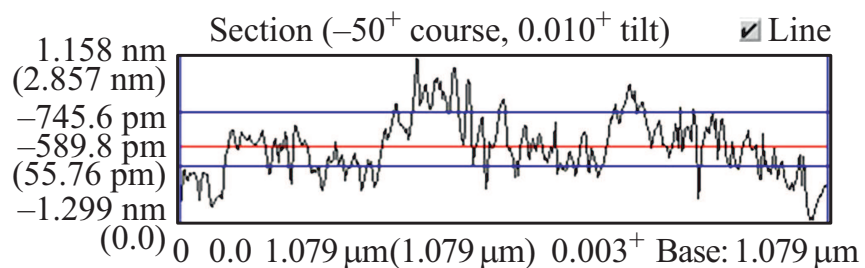

Rms rough. $\left(R_{q}\right): 529.8 \mathrm{pm} \quad$ Mean width $\left(S_{m}\right): 32.56 \mathrm{~nm}$ Sect. smooth: 1 . Mean rough. $\left(R_{a}\right): 419.1 \mathrm{pm} \quad$ Localwidth $(S): 13.69 \mathrm{~nm} \quad$ Hist. smooth: 1 . 10 ptmean. $\left(R_{z}\right): 1.748 \mathrm{~nm} \quad$ Rms slope $\left(D_{q}\right): 5.052^{+}$

$\operatorname{Maxheight}\left(R_{\max }\right): 2.857 \mathrm{~nm}$ Mean slope $\left(D_{a}\right): 3.629^{+}$

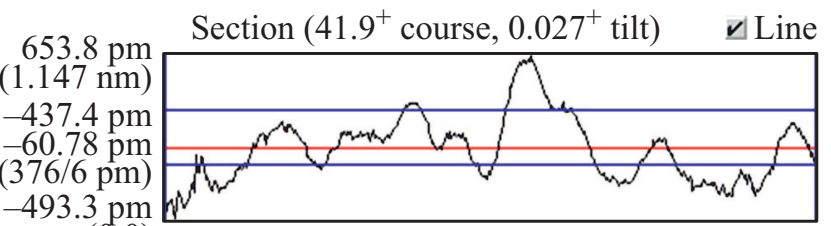

(0.0) $00.01 .240 \mu \mathrm{m}(1.240 \mu \mathrm{m}) 0.017^{+}$Base: $1.240 \mu \mathrm{m}$

Rms rough. $\left(R_{q}\right): 227.8 \mathrm{pm} \quad$ Mean width $\left(S_{m}\right): 159.7 \mathrm{~nm}$ Sect. smooth: 1 」 Mean rough. $\left(R_{a}\right): 185.0 \mathrm{pm}$ Localwidth $(S): 13.80 \mathrm{~nm}$ Hist. smooth: 1 . 10 pt mean. $\left(R_{z}\right): 462.2 \mathrm{pm} \quad$ Rms slope $\left(D_{q}\right): 0.704^{+}$

$\operatorname{Maxheight}\left(R_{\max }\right): 1.147 \mathrm{~nm}$ Mean slope $\left(D_{a}\right): 0.513^{+}$

Рис. 3. Статистический анализ СТM $(a)$ и $\mathrm{ACM}(b)$ изображений рельефа поверхности пленки Мо толщиной $19 \mathrm{~nm}$, произведенный программой микроскопа СММ-2000 по стандартным методикам.

Таблица 1. Результаты статистического анализа пленок Мо разных толщин

\begin{tabular}{c|c|c|c|c|c|c}
\hline Параметры & \multicolumn{2}{|c|}{$S_{q}, \mathrm{~nm}$} & \multicolumn{2}{c|}{$S m, \mathrm{~nm}$} & \multicolumn{2}{c}{$S, \mathrm{~nm}$} \\
\hline Методы & СТМ & ACM & СТМ & ACM & СТM & ACM \\
\hline Толщина 3 nm & 0.342 & 0.099 & 36.2 & 77.4 & 9.73 & 15.1 \\
Толщина 6 nm & 0.184 & 0.085 & 27.4 & 87.3 & 14.4 & 14.7 \\
Толщина 19 nm & 0.529 & 0.227 & 32.5 & 159.7 & 13.7 & 13.8
\end{tabular}

нения, $S_{q}$ для СТМ данных в $2.2-3.5$ раза превышает таковую для АСМ. Латеральный размер особенностей рельефа поверхности $\mathrm{Sm}$ на большом масштабе - наоборот, в $2-5$ раз меньше, а на малом масштабе $(S)$ он примерно одинаков.

Полученные результаты можно объяснить тем, что при достаточно больших отклонениях рельефа по вертикали от среднего значения, зависимость тока от высоты подъема иглы над поверхностью в СТМ измерениях уже значительно отличается от линейной. Тем самым СТМ метод дает более высокие значения среднеквадратичной шероховатости $S_{q}$.

СТМ метод дает также меньшие значения латерального размера особенностей рельефа поверхности $\mathrm{Sm}$. Это, по всей видимости, связано с тем, что из-за нелинейности зависимости тока от высоты подъема иглы малые особенности рельефа начинают проявляться в СТМ изображении сильнее, чем в АСМ. При этом появляются пересечения средней линии рельефа (рис. 3) малыми особенностями $S$. Также АCM кантилевер сглаживает малые особенности из-за конечной величины диаметра острия.

Был проведен также фурье-анализ поверхности образцов. Латеральные размеры больших и малых особенностей рельефа поверхности были определены по положению первого и второго пиков в фурье-спектре. В табл. 2 сведены значения периодов больших и малых особенностей рельефа пленок, определенные с помощью фурье-анализа, а также корреляционной длины в зависимости от толщины пленки. Как видно из таблицы, величины больших и малых периодов для СТМ и ACM данных близки, а корреляционные длины - это примерно среднее значение от этих двух величин. Таким образом, фурье-анализ подтверждает сходство между результатами измерений двумя разными методами, которое также наблюдается визуально (рис. 1).

На рис. 4 показана зависимость среднеквадратичной шероховатости поверхности пленок Мо от их толщины, полученная нами ранее [3] по результатам АСМ и СТМ измерений, с добавлением новых значений из измерений, представленных на рис. 1. Как видно из рисунка, погрешность АСМ измерений довольно высока. Это связано во многом как со спецификой АСМ измерений (как-то сбоями в контрасте изображения в процессе работы), так и с использованием подложек с разными шероховатостями. Оценки погрешностей были проведены в предыдущей работе [3]. Новые АСМ измерения (на рис. 4 обозначенные пятиугольниками), проведенные в настоящей работе, имеют меньшую погрешность, по-

Таблица 2. Результаты фурье-анализа пленок Мо разных толщин

\begin{tabular}{c|l|l|c|c|c|c}
\hline Параметры & \multicolumn{2}{|c|}{$L_{c}, \mathrm{~nm}$} & \multicolumn{2}{c|}{$S m, \mathrm{~nm}$} & \multicolumn{2}{c}{$S, \mathrm{~nm}$} \\
\hline \multicolumn{1}{c|}{ Методы } & СТМ & ACM & СТM & АCM & СТМ & ACM \\
\hline Толщина 3 $\mathrm{nm}$ & 61.64 & 47 & 85.5 & 87.09 & 28.91 & 22.35 \\
Толщина 6 $\mathrm{nm}$ & 23.56 & 49.6 & 95.47 & 140.4 & 32.88 & 29.99 \\
Толщина $19 \mathrm{~nm}$ & 89.6 & 219 & 272.2 & 287.6 & 41.75 & 40.99
\end{tabular}


скольку перед напылением пленок рельеф поверхности подложек измерялся с помощью АСМ и выбирались подложки с малой шероховатостью поверхности, а также использовались более острые кантилеверы. Несмотря на значительную погрешность АСМ измерений, видно, что результаты СТМ измерений выходят за пределы погрешности данных АCM измерений и дают более высокие значения шероховатости. На это было указано в работе [3], и этот результат повторился при более точных измерениях, проведенных в настоящей работе.

\section{4. Численное моделирование}

Как показывают экспериментальные данные (рис. 4), с увеличением толщины среднеквадратичная шероховатость пленок возрастает. Численное моделирование, согласно уравнению (4), в пренебрежении флуктуационным членом и поверхностным натяжением показывает, что в таком приближении расчетная шероховатость падает. Член в уравнении (4), описывающий поверхностное натяжение, не может привести к росту шероховатости, он может только сглаживать поверхность [12], следовательно, для соответствия эксперименту необходимо добавить флуктуационный член.

В этом смысле Мо, как тугоплавкий металл, является хорошим модельным материалом, чтобы исключить влияние поверхностной диффузии, приводящей к учету производных четвертого порядка в уравнении модели, поскольку диффузионная подвижность атомов Мо при используемых температурах будет крайне малой в сравнении с обычно изучаемыми материалами. Это хорошо подтверждается, например, сравнением роста пленок $\mathrm{Fe}$ и Мо. Так, для железа получить пленки с малой шероховатостью весьма проблематично из-за происходящего, так называемого механизма $3 d$ роста пленок Вольмера-Вебера [17], связанно-

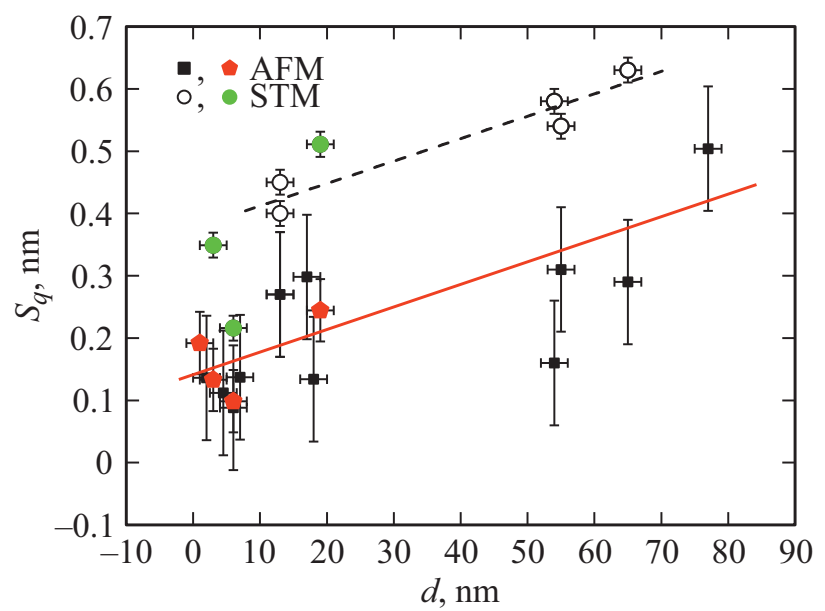

Рис. 4. Зависимость среднеквадратичной шероховатости поверхности пленок Мо от их толщины по результатам АСМ (квадраты) и СТМ (кружки) измерений. Заполненные пятиугольники и кружки - новые АСМ и СТМ измерения. го с разницей поверхностных энергий $\left(\gamma_{\mathrm{Fe}}=2.4 \mathrm{~J} / \mathrm{m}^{2}\right.$, $\left.\gamma_{\mathrm{Al}_{2} \mathrm{O}_{3}}=1.2 \mathrm{~J} / \mathrm{m}^{2}[18]\right)$ сапфира и железа. Для пары сапфир-молибден эта разница в поверхностных энергиях еще больше $\left(\gamma_{\text {мо }}=3.4 \mathrm{~J} / \mathrm{m}^{2}[19]\right)$, что должно бы привести к еще более значительному $3 d$ росту. Тем не менее при используемых температурах диффузия атомов Мо подавлена, поэтому получение относительно гладких пленок Мо не является такой большой проблемой, как для того же железа (см. [20] и ссылки в ней).

В настоящей работе было вначале проведено численное моделирование эволюции рельефа поверхности пленок, согласно уравнению (4), в пренебрежении поверхностным натяжением. Как уже было сказано, исходная шероховатость подложек сапфира составляла около $0.2 \mathrm{~nm}$.

Моделирование производилось по следующему алгоритму. Брался некоторый небольшой интервал времени $t$, такой, что $c t \approx 5 \mathrm{~nm}$. Для этого интервала с помощью принципа Олейник-Лакса вычислялось решение уравнения (4) без члена с поверхностным натяжением и флуктуационного члена и с начальным условием в виде экспериментального АСМ скана подложки сапфира. Затем к полученному решению добавлялись флуктуации в виде шума с некоторой дисперсией $\sigma^{2}$, гауссовым распределением по $z$ и некоррелированного в плоскости пленки. Такое решение с шумом бралось в качестве начального условия для того, чтобы получить решение на следующем таком же интервале времени и т.д. Было найдено, что среднеквадратичное отклонение (корень из дисперсии) шума на таком интервале времени должно быть не меньше, чем 1/20 от максимального значения высоты рельефа подложки, т. е. около $0.1 \mathrm{~nm}$. При меньших значениях этого параметра расчет не дает увеличения шероховатости поверхности в процессе роста.

Далее был реализован второй способ решения уравнения (3) согласно формуле (12). Значение параметра $v$ было выбрано $0.1 c$ из соображений, что оно должно быть малым по сравнению со скоростью роста. Для выбранного интервала времени производилось численное интегрирование (12) по пространственным координатам. Результат подставлялся в формулу (10) и добавлялся шум с выбранной ранее амплитудой.

Результаты расчетов представлены на рис. 5. Из рис. 5, $a$ видно, что шероховатость поверхности увеличивается, что соответствует эксперименту. Добавление поверхностного натяжения уменьшает значение шероховатости, однако тенденция ее роста сохраняется. Фрактальная размерность (рис. $5, b$ ) остается приблизительно постоянной, что тоже подтверждается экспериментально, причем добавление члена поверхностного натяжения не повлияло на ее расчетные значения, а корреляционная длина уменьшается (рис $5, c)$.

Далее был проведен анализ соответствия модели КПЖ эксперименту для разных значений параметров $v$ и $\sigma$. На рис. 6 показана область этих параметров, внутри которой модель дает увеличение шероховатости 
с толщиной пленки при данной шероховатости подложки (закрашенная область). Граница этой области получилась близкой к прямой $\sigma \approx \sigma_{0}+0.1 v / c$, которая пересекает ось ординат при $\sigma=\sigma_{0} \approx 0.1 \mathrm{~nm}$. Увеличение шероховатости, согласно модели, имеет место в достаточно большом диапазоне $v$ и $\sigma$. Однако корреляционная длина при значениях $\sigma$ более $0.15 \mathrm{~nm}$ уже выходит на
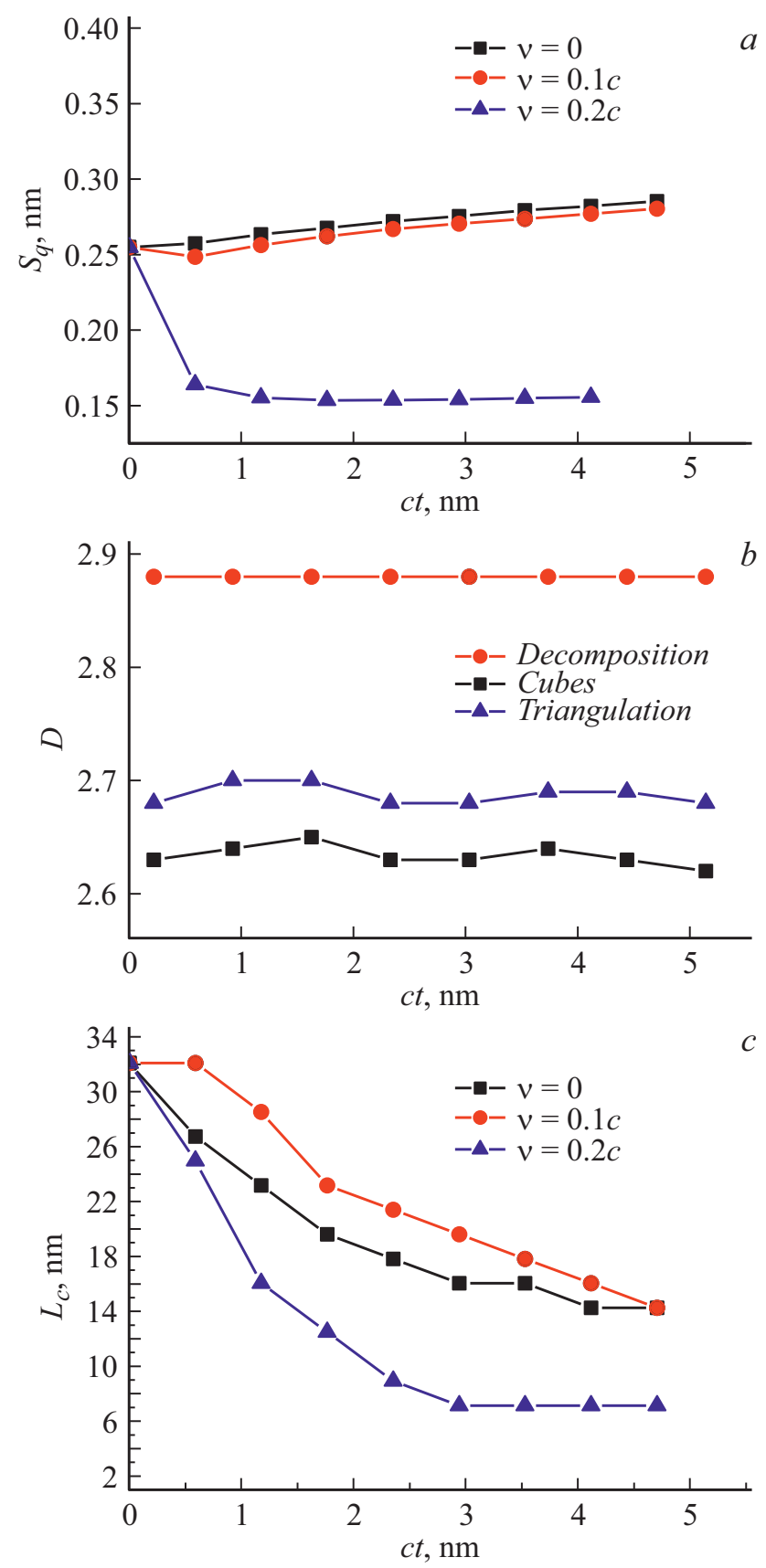

Рис. 5. Зависимости среднеквадратичной шероховатости для расчетов без поверхностного натяжения и с добавлением поверхностного натяжения $v=0.1$ и $0.2 c(a)$, фрактальной размерности $(b)$, полученной методами подсчета числа кубов (квадраты), триангуляции (треугольники) и декомпозиции (кружки), а также корреляционной длины (c) рельефа поверхности по результатам численного моделирования.

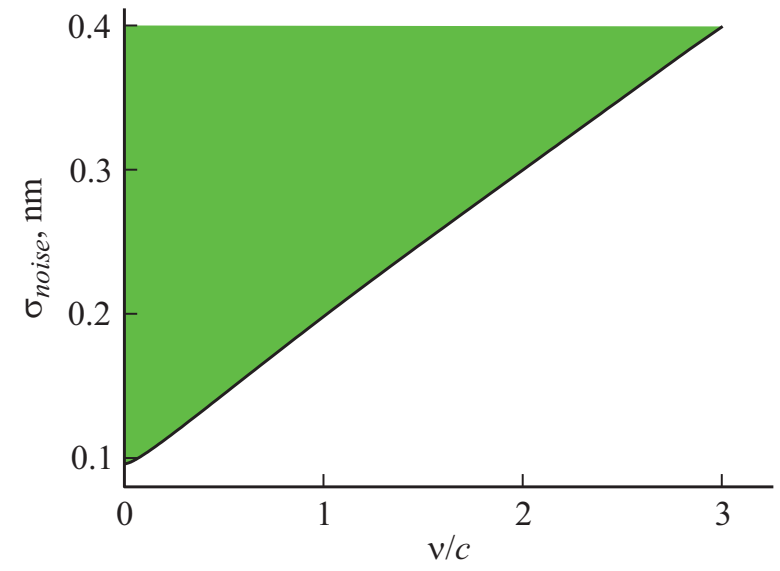

Рис. 6. Область параметров $v$ и $\sigma$, внутри которой модель КПЖ дает увеличение шероховатости с толщиной пленки.

минимальное значение, определяемое шумом, что не согласуется с экспериментом.

Таким образом, результаты численного моделирования качественно совпадают с экспериментальными в треугольной области значений параметров $\sigma \approx 0.1-0.15 \mathrm{~nm}$ и $0 \leq v \leq 10 c(\sigma-0.1)$. Вне этой области значений параметров среднеквадратичная шероховатость не увеличивается с толщиной. Минимальная пороговая амплитуда флуктуаций, при которой модель КПЖ адекватно описывает рост пленок Мо оказывается равной $0.1 \mathrm{~nm}$.

\section{5. Обсуждение}

Показано, что полученные пленки Мо, в том числе сверхтонкие, устойчивы к процессам окисления, что позволяет проводить измерения их морфологии поверхности в течение дней. Равномерное окисление пленки не сильно влияет на ее рельеф, измеряемый методом АСМ. Однако на СТМ изображения оно влияет сильнее. Можно предположить, что процесс окисления пленки описывается той же самой моделью КПЖ. В пренебрежении поверхностным натяжением модель дает разрыв пространственных производных рельефа поверхности в ямках из-за набегания друг на друга двух фронтов роста [14]. Если же принять во внимание член поверхностного натяжения, то поверхность оксида в ямках заглаживаться. Это способствует накоплению там большего количества материала, чем на бугорках. Поскольку зависимость туннельного тока от толщины диэлектрика экспоненциальная, даже небольшое изменение толщины оксида должно приводить к сильному увеличению контраста в СТМ изображении, что, по всей видимости, мы и наблюдаем. 


\section{Заключение}

Сравнение измерения морфологии поверхности методами СТМ и АСМ сверхтонких эпитаксиальных пленок Мо на $R$-плоскости сапфира показало, что метод CТМ дает более высокие значения среднеквадратичной шероховатости по сравнению с АСМ. Использование подложек с малой шероховатостью дает тот же результат, как и в случае использования подложек с произвольной шероховатостью. Было найдено, что процессы окисления пленок Мо происходят крайне медленно, что позволяет проводить их исследования в течение разумных промежутков времени (нескольких дней). Показано, что для адекватного соответствия экспериментальным результатам по увеличению шероховатости пленок Мо в модели эволюции рельефа поверхности пленок Кардара-Паризи-Жанга необходимо учесть флуктуационный член, минимальная величина которого оценена как 1/20 от максимального значения высоты рельефа подложки, что составляет около $0.1 \mathrm{~nm}$. Модель описывает экспериментальные результаты в треугольной области значений параметров среднеквадратичного отклонения шума $\sigma \approx 0.1-0.15 \mathrm{~nm}$ и поверхностного натяжения $0 \leq v \leq 10 c(\sigma-0.1)$.

\section{Финансирование работы}

Работа выполнена при поддержке Государственного задания № 075-00355-21-00.

\section{Конфликт интересов}

Авторы заявляют, что у них нет конфликта интересов.

\section{Список литературы}

[1] P. Kapur, J.P. McVittie, K.C. Saraswat. IEEE Trans. Electron Dev. 49, 590 (2002). DOI: 10.1109/16.992867

[2] C.Y. Pan, A. Naeemi. IEEE Electron Dev. Lett., 35, 250 (2014). DOI: 10.1109/LED.2013.2291783

[3] Л.А. Фомин, И.В. Маликов, В.А. Березин, А.В. Черных, А.Б. Логинов, Б.А. Логинов. ЖТФ, 90 (11), 1830 (2020). [L.A. Fomin, I.V. Malikov, V.A. Berezin, A.V. Chernykh, A.B. Loginov, B.A. Loginov. Tech. Phys., 65 (11), 1748 (2020).] DOI: 10.21883/JTF.2020.11.49970.110-20

[4] R.C. Munoz, G. Vidal, G. Kremer, L. Moraga, C. Arenas, A. Concha. J. Phys.: Condens. Matter., 12, 2903 (2000). DOI: $10.1088 / 0953-8984 / 12 / 13 / 302$

[5] R.C. Munoz, G.Vidal, M. Mulsow, J.G. Lisoni, C. Arenas, A. Concha, F. Mora, R. Espejo, G. Kremer, L. Moraga, R. Esparza, P. Haberle. Phys. Rev. B, 62, 4686 (2000). DOI: 10.1103/PhysRevB.62.4686

[6] M. Kardar, G. Parisi, Y.C. Zhang. Phys. Rev. Lett., 56, 889 (1986). DOI: 10.1103/PhysRevLett.56.889

[7] K.A. Takeuchi. Physica A, 504, 77 (2018). DOI: $10.1016 /$ j.physa.2018.03.009

[8] M. Gubinelli, N. Perkowski. Comm. Math. Phys., 349, 165 (2017). DOI: 10.1007/s00220-016-2788-3
[9] A. Krajenbrink, P. Le Doussal. Phys. Rev. E, 96, 020102 (2017). DOI: 10.1103/PhysRevE.96.020102

[10] B. Meerson, E. Katzav, A. Vilenkin. Phys. Rev. Lett., 116, 070601 (2016). DOI: 10.1103/PhysRevLett.116.070601

[11] J. Villain. J. Phys., I 1, 19 (1991). DOI: 10.1051/jp1:1991114

[12] A.-L. Barabasi, H.E. Stanley. Fractal concepts in surface growth (Cambridge University Press, 1995), 388 p.

[13] И.В. Воротынцев, А.Э. Рассадин, Л.А. Фомин, И.В. Маликов. Поверхность. Рентген., синхротр. и нейтрон. исслед., 4, 90 (2021). [I.V. Vorotyntsev, A.E. Rassadin, L.A. Fomin, I.V. Malikov. J. Surf. Investigation: X-ray, Synchrotron and Neutron Techniq., 15, 389 (2021).] DOI: $10.1134 / \mathrm{S} 1027451021020348$

[14] С.Н. Гурбатов, О.В. Руденко, А.И. Саичев. Волны и структуры в нелинейных средах без дисперсии. Приложения к нелинейной акустике (Физматлит, М., 2008), 496 с.

[15] О.А. Олейник. УМН, 12 (3), 3 (1957).

[16] I.V. Malikov, L.A. Fomin, V.A. Berezin. Ferroelectrics, 559 (1), 150 (2020). DOI: 10.1080/00150193.2020.1722017

[17] К. Оура, В.Г. Лифшиц, А.А. Саранин, А.В. Зотов, М. Катаяма. Введение в физику поверхности (Наука, М., 2006), $490 \mathrm{c}$.

[18] N. Eustathopoulos, M.G. Nicholas, B. Drevet. Wettability at High Temperatures (Pergamon Materials Series, 1999), v. 3.

[19] U. May, R. Calarco, J.O. Hauch, H. Kittur, M. Fonine, U. Rudiger, G. Güntherodt. Surf. Sci., 489, 144 (2001). DOI: $10.1016 / \mathrm{S} 0039-6028(01) 01172-4$

[20] L.A. Fomin, I.V. Malikov, S.V. Pyatkin, G.M. Mikhailov. J. Magn. Magn. Mater., 322, 851. DOI: $10.1016 /$ j.jmmm.2009.11.016 